\title{
Specificity of Fur Binding to the Oxidative Stress Response Gene Promoter in the Facultative Anaerobic Archaeon Thermoplasma volcanium
}

\author{
Hikari Minoshima, ${ }^{a}$ Yu Ikeda, ${ }^{a}$ Miki Fujii,${ }^{a}$ Mai Satoh, ${ }^{a}$ Tomoaki Ishikawa, ${ }^{a}$ Youichi Itoh, ${ }^{b}$ \\ Yoshie Kawashima-Ohya ${ }^{c}{ }^{\text {Koji Tomobe }}{ }^{d}{ }^{\text {Yoshiaki Omata },{ }^{a} \text { and Tsuyoshi Kawashima*, } a}$ \\ ${ }^{a}$ Department of Molecular Biology, Yokohama College of Pharmacy; ${ }^{b}$ Department of Kampo Therapy, Yokohama \\ College of Pharmacy; ${ }^{c}$ Department of Immunology, Yokohama College of Pharmacy; and ${ }^{d}$ Department of \\ Pathophysiology, Yokohama College of Pharmacy; 601 Matano-cho, Totsuka-ku, Yokohama 245-0066, Japan. \\ Received August 4, 2013; accepted December 13, 2013
}

\begin{abstract}
The genome of the facultative anaerobic thermoacidophilic archaeon Thermoplasma volcanium contains the open-reading frames (ORFs) tvsod and tvogg, which are predicted to encode a putative superoxide dismutase and an 8-oxoguanine DNA glycosylase, respectively. Tvsod is immediately upstream of tvogg, and these two ORFs are aligned in a head-to-tail manner in a single operon. A previous study showed that $T$. volcanium contains an ORF (TVN0292) encoding the ferric uptake regulator (Fur) and that the T. volcanium Fur protein (TvFur) binds to its own promoter in a metal-dependent manner in vitro. Here, we demonstrated that TvFur also binds to the tvsod-tvogg promoter and determined the TvFur-binding sequences in the tvsodtvogg promoter by DNaseI footprinting analysis. These results suggest that Fur is required for resistance against reactive oxygen species in this facultative anaerobic archaeon.
\end{abstract}

Key words archaea; ferric uptake regulator (Fur); superoxide dismutase gene promoter

Archaea $^{1)}$ is a taxon that constitutes a kingdom of life. Almost two-thirds of the archaea are thermophiles or hyperthermophiles, ${ }^{2)}$ and most of them are strict anaerobes. ${ }^{3)}$ The proteins encoded by their genomes are heat-stable, and some of them are utilized in bioscience research, for example, polymerase chain reaction (PCR) employs thermophilic DNA polymerases. Therefore, research on additional proteins encoded by thermophilic and hyperthermophilic archaea could likely be beneficial.

In general, eukarya cannot survive in the absence of oxygen because they require molecular oxygen to transfer the hydrogen ions (electrons) to that are produced by oxidation of nutrients through the electron transport pathway to generate energy. Nevertheless, reactive oxygen species (ROS) generated by these electron transport pathways are toxic because macromolecules such as proteins and DNA are readily oxidized by ROS. This explains why internal injuries from oxidative-stress are inevitable for organisms that use oxygen as a terminal electron acceptor. Therefore, establishing defense systems against this kind of oxidation is necessary for any organisms that live in an aerobic environment.

Among the vast number of macromolecules that are oxidized by ROS, DNA oxidation may result in the most serious consequences. In particular, the generation of 8-oxo-7,8dehydro-2'-deoxyguannosine $(8-0 x o G)$ by oxidation of guanine is the most frequently observed lesion in DNA. ${ }^{4,5)} 8$-oxoG has been shown to form a stable base pair with both cytosine and adenine; therefore, the 8-oxoG: A pair can undergo transversion from $\mathrm{G}: \mathrm{C}$ to $\mathrm{T}: \mathrm{A}$ after replication. ${ }^{6,7)}$ To prevent this transversion mutation, organisms have evolved base excision repair pathways that utilize a specific DNA glycosylase.

In anaerobic archaea, an enzyme called superoxide reductase (SOR) has evolved as a defense system that removes ROS without generating molecular oxygen. ${ }^{8)}$ Most organisms

The authors declare no conflict of interest. that live in aerobic environments also possess another enzyme to detoxify ROS, superoxide dismutase (SOD). Under aerobic conditions, some archaea adopt oxygen as terminal electron acceptor. ${ }^{3)}$ Thermoplasma volcanium is one of these aero-anaero facultative archaea $^{9)}$ that has a putative SOD gene (TVN0061, designated tvsod). The $T$. volcanium genome also encodes a putative 8-oxoG DNA glycosylase (TVN0062, designated tvogg) that removes the oxidized guanines as well as a uracil DNA glycosylase (TVN0827) that removes the uracils generated by deamination of cytosine, ${ }^{10}$ and an AP endonuclease (TVN0046 and TVN0971) that restores an apurinic/ apyrimidinic site. ${ }^{11)}$ In the genome of $T$. volcanium, tvogg is located in an operon with tvsod, and these two genes constitute a single transcription unit (Toda et al., unpublished). This gene configuration makes it possible to rapidly respond to oxidative stress.

In numerous bacteria, the ferric uptake regulator (Fur) is a transcriptional regulator of genes encoding proteins that are involved in iron homeostasis and protection from the effects of iron toxicity, including oxidative stress under aerobic conditions. ${ }^{12)} T$. volcanium contains a gene encoding Fur (TVN0292, designated tvfur), and TvFur, the T. volcanium Fur protein, binds to its own promoter region in vitro. ${ }^{13)}$ In this study, we investigated the binding specificity of TvFur to the tvsod-ogg operon promoter by electrophoretic mobility shift assay (EMSA), and determined the TvFur binding sequence in this promoter by DNaseI footprinting analysis. TvFur recognized a TATA-box like TA repeat sequences.

\section{MATERIALS AND METHODS}

Strains Thermoplasma volcanium GSS1 (NBRC 15438) was used as a source of whole genomic DNA. Cloning of the gene encoding TvFur, as well as the overexpression and purification of the TvFur protein have been described previously. ${ }^{13)}$

Electrophoretic Mobility Shift Assays (EMSA) A 204- 
bp DNA fragment including the tvsod-ogg promoter and a portion of its coding region (from -132 to +72 ; designated as $5^{\prime}$ tvsod-ogg), corresponding to bases 62122-62325 of $T$. volcanium (GenBank accession no. NC_002689.2) was amplified from genomic DNA by PCR with the primers psod 5' (5'-AAC AGA ATT CAT CGC CTC CAG AG-3') and psod 3' (5'-TAT CTG TAC ATC TGA ATT CCT TC-3') carrying EcoRI restriction sites. The 5'tvsod-ogg fragment was cloned into the pGEM-T Easy vector (Promega), and then digested with EcoRI. The digested 5' tvsod-ogg fragment was gel purified and subcloned into pUC119 (TaKaRa) to generate pUC119-5' tvsod-ogg. For EMSA, a DNA fragment containing $5^{\prime}$ tvsod-ogg was prepared by PCR amplification of pUC119-5' tvsod-ogg with the primer pair M13-20-FAM (5'-6-FAM-GTT TTC CCA GTC ACG AC-3') and M13rev (5'-CAG GAA ACA GCT ATG AC- $\left.3^{\prime}\right)$. As a negative control, 50S ribosomal protein L3P gene (TVN0324 rpl3p, first gene of ribosomal operon corresponded with 332101 to 332360 of NC_002689.2) was prepared. To determine the minimal recognition region for TvFur binding, a series of oligonucleotides containing TvFur binding sequences or those with several bases substitutions were prepared. TvFur binding sequences were determined by the DNaseI footprinting analysis described below. The $5^{\prime}$ FAM labeled or non-labeled double-stranded DNA for EMSA was generated by annealing complementary oligonucleotides by incubation at $95^{\circ} \mathrm{C}$ for $5 \mathrm{~min}$ and then cooling to room temperature. EMSA was performed with $0.2 \mu \mathrm{M}$ or $0.4 \mu \mathrm{M}$ of DNA under essentially the same conditions as previously described $^{13)}$ except electrophoresis buffer containing $1 \mathrm{~mm}$ dithiothreitol (DTT). The DNA bands were detected and analyzed with a Pharos FX molecular imager and Quantity One imaging software (Bio-Rad), and the dissociation constant $\left(K_{\mathrm{d}}\right)$ was calculated. ${ }^{14)}$

DNaseI Footprinting Analysis DNaseI footprinting analysis was performed according to the method described by Riboulet-Bisson et al. ${ }^{15)}$ with some modification. The $5^{\prime}$ tvsod-ogg fragment was generated by PCR with pUC119-5' tvsod-ogg as a template and a $\mathrm{D} 4$ dye end-labeled primer set of M13-20 (5'-D4-GTA AAA CGA CGG CCA GT-3') and M13rev (5'-D4-GGA AAC AGC TAT GAC CAT G-3'). The binding reaction contained 1.0 pmol of end-labeled DNA fragment and various amounts of TvFur protein $(0.75-1.25 \mathrm{pmol})$ and was incubated at room temperature for $10 \mathrm{~min}$ in $35 \mu \mathrm{L}$ of $40 \mathrm{~mm} \mathrm{KCl,} 1 \mathrm{~mm}$ DTT, $100 \mu \mathrm{M} \mathrm{MnCl}_{2}, 1 \mu \mathrm{g} / \mathrm{mL}$ bovine serum albumin (BSA), $1 \mu \mathrm{g} / \mathrm{mL}$ poly $(\mathrm{d} I-\mathrm{d} C), 5 \%$ glycerol, and $10 \mathrm{~mm}$ Tris- $\mathrm{HCl}, \mathrm{pH}$ 8.0. The product was then digested with $0.01 \mathrm{U}$ of freshly diluted RNase free DNaseI (Roche) in the same buffer for $5 \mathrm{~min}$ at room temperature. The reaction was terminated by the addition of $5 \mu \mathrm{L}$ of $250 \mathrm{~mm}$ ethylenediaminetetraacetic acid (EDTA). The samples were analyzed with the CEQ8800 sequencing apparatus (Beckman Coulter). Determination of the DNA sequence of the protected region was performed according to a previously described method. ${ }^{13)}$

\section{RESULTS AND DISCUSSION}

TvFur Specifically Binds to the tvsod-ogg Promoter Region In the TvFur 5' tvsod-ogg DNA binding experiments, the bound fraction of the FAM-labeled $5^{\prime}$ tvsod-ogg DNA fragment increased as the amount of TvFur protein was raised from $1.0 \mu \mathrm{M}$ to $4.0 \mu \mathrm{M}$ (Fig. 1A, lanes 2-5). Bound TvFur
$\mathbf{A}$

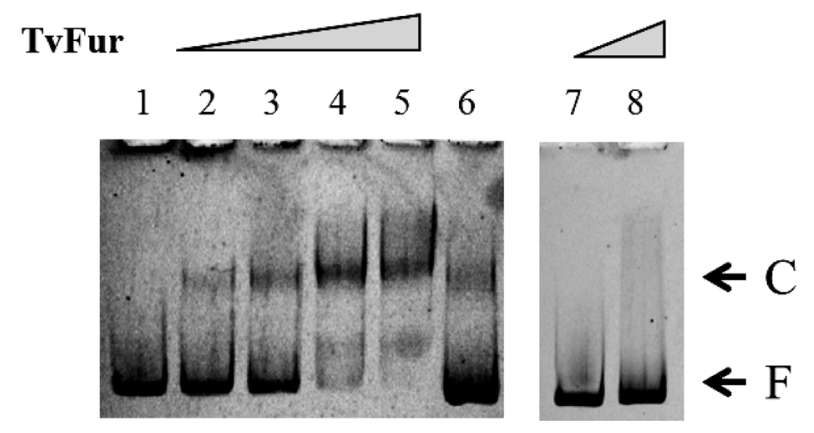

$\mathbf{B}$

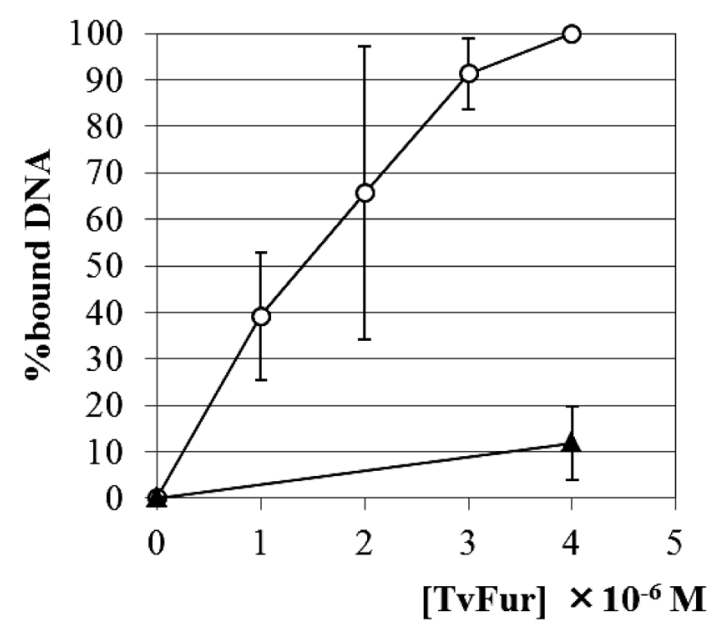

Fig. 1. Electrophoretic Mobility Shift Assay (EMSA) with $0.2 \mu \mathrm{M}$ of FAM-Labeled tvsod-ogg Promoter DNA (Lanes 1-6) or the Promoter DNA of TVN0324 rpl3p, First Gene of Ribosomal Operon (Lanes 7 and 8) as a Negative Control Incubated with TvFur in the Presence of $100 \mu \mathrm{M}$ $\mathrm{MnCl}_{2}$

(A) Lanes 1 and 7, no TvFur protein; lanes 2-5 contained 1.0, 2.0, 3.0 and $4.0 \mu \mathrm{M}$ of TvFur protein, respectively. Lane 6 contained $0.2 \mu \mathrm{M}$ of FAM-labeled $t v \operatorname{sod}$-ogg promoter DNA, $1.0 \mu \mathrm{m}$ of non-labeled tvsod-ogg promoter DNA and $4.0 \mu \mathrm{m}$ of TvFur protein. $\mathrm{C}$ and $\mathrm{F}$ indicate TvFur bound and free (unbound) promoter DNA, respectively. (B) Binding profile of TvFur to $0.2 \mu \mathrm{M}$ of $t v s o d$-ogg promoter DNA. The TvFur concentration varied from 0 to $4.0 \mu \mathrm{M}$ as indicated above. Error bars represent the S.D. of three independent experiments. $\bigcirc$, TvFur binding to the $t v s o d$ ogg promoter DNA; $\boldsymbol{\Delta}$, TvFur binding to the rpl3p gene promoter.

competed with fivefold excess of non-labeled promoter DNA (Fig. 1A, lane 6). The binding of TvFur to the 5' tvsod-ogg DNA fragment was only observed in the presence of $100 \mu \mathrm{M}$ $\mathrm{Mn}^{2+}$, while no binding was observed in metal ion-free binding buffer (data not shown). No unbound DNA was observed when the EMSA included $4.0 \mu \mathrm{M}$ of TvFur (Fig. 1A, lane 5). TvFur scarcely bind to the ribosomal protein L3 encoding gene promoter (Fig. 1A, lane 8), demonstrating that TvFur specifically binds to the tvsod-ogg promoter region. From the EMSA results, the dissociation constant $\left(K_{\mathrm{d}}\right)$ was calculated as $1.3 \times 10^{-6} \mathrm{M}$ (Fig. 1B). The dissociation constant for TvFur binding to its own promoter was approximately same value, ${ }^{13)}$ however that the constant for binding to the tvhemB promoter was $37 \%$ of this value (data not shown). These results indicate that TvFur binds to the former two promoters with higher affinities than to the $t$ them $B$ promoter region. Therefore, TvFur could robustly regulate the expression of tvsod-ogg genes.

Estimation of the Essential Recognition Region for 
A

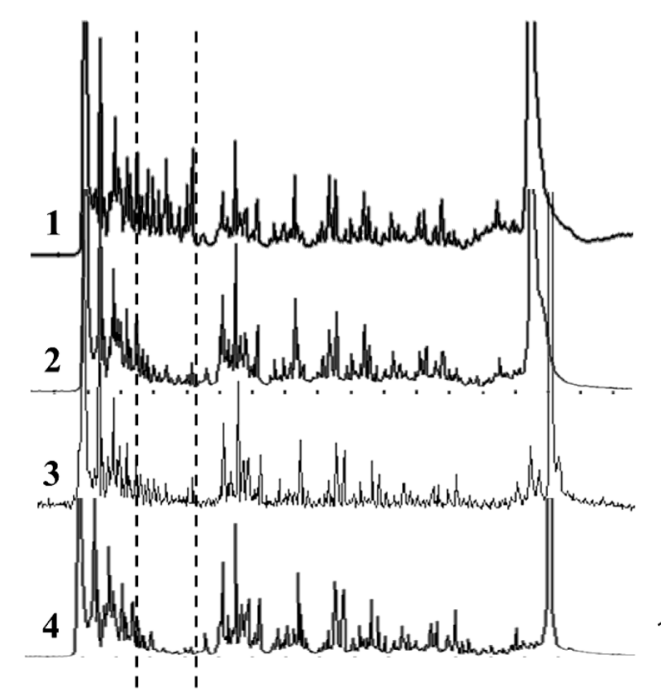

B

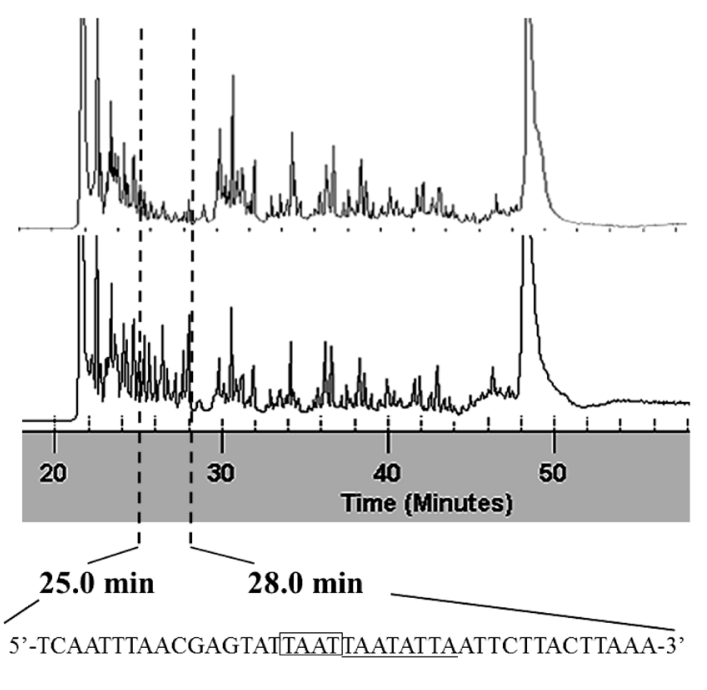

Fig. 2. Identification of the TvFur Binding Region in the tvsod-ogg Promoter by DNaseI Footprinting Analysis

Fluorescence intensity (fragment abundance) is shown on the $y$ axis and elution position (size) is shown on the $x$ axis. (A) Full length electropherograms of 1.0 pmol of 5' tvsod-ogg DNA after DNase I footprinting analysis without TvFur (1) or with 0.75 pmol (2), 1.0 pmol (3), and 1.25 pmol (4) of TvFur. The elution position between two dotted lines represents the region protected from DNaseI digestion by TvFur binding. (B) Full length electropherograms after DNaseI footprinting analysis performed without (lower panel) or with $1.25 \mathrm{pmol}$ of TvFur (upper panel). The DNA region corresponding to $25-28$ min after start (between the left and right dotted lines) was the region protected from DNaseI digestion by TvFur binding. The TvFur binding sequence identified by footprinting analysis is indicated at the bottom. The putative BRE and TATA-box are boxed and underlined, respectively.

TvFur Binding To determine the exact location where TvFur binds to the tvsod-ogg promoter, DNaseI footprinting was performed (Fig. 2). The protected regions in a DNaseI footprinting reaction with $1.0 \mathrm{pmol}$ of $5^{\prime}$ tvsod-ogg DNA and 0.75 pmol of TvFur were not changed by increasing the amount of TvFur to 1.25 pmol. (Fig. 2A). The DNA sequence of the protected region were corresponded to from $25.0 \mathrm{~min}$ to $28.0 \mathrm{~min}$ on the electropherogram (Fig. 2B). This region corresponded to the 41-bp sequence of 5'-TCA ATT TAA CGA GTA TTA ATT AAT ATT AAT TCT TAC TTA AA-3' (Fig. $2 \mathrm{~B})$, which extended to positions -92 to -51 relative to the translational start site of $t v s o d$. These results indicated that the TvFur binding region included the TATA-box or was in the vicinity of the TATA-box in the tvsod-ogg promoter. The TvFur binding sequence in the tvfur promoter region includes the 30-bp sequence 5'-GTT ATT ATG TTT ATA T AT TAA TTA GTA CAA-3' when 0.8 pmol of TvFur and 1 pmol of DNA was used in a DNaseI footprinting assay. In contrast to the results obtained for the tvsod-ogg promoter, the protected region of the tvfur promoter extended downstream towards the start codon depending on the amount of TvFur. ${ }^{13)}$ The $T$. volcanium consensus BRE and TATA-box sequences are 5'-AAAA-3' and $5^{\prime}$-TTTATATA-3', respectively, ${ }^{16}$ ) and both of them are consistent with the archaeal consensus sequences $5^{\prime}$-WAAW-3' (BRE) and 5'-YTTWWAWW-3' (TATA-box). ${ }^{17)}$ The putative BRE of the tvsod-ogg promoter, 5'-TAAT-3', was consistent with the consensus archaeal BRE sequence, whereas the putative TATA-box of the tvsod-ogg promoter, 5'-TAATATTA-3', differed from the archaeal consensus TATA-box sequence. This divergence between the putative TATA-box of the tvsodogg promoter and the archaeal consensus TATA-box might enable TvFur to regulate the transcriptional level of $t v s o d$-ogg. To determine the precise region of where TvFur interacts with the tvsod-ogg promoter in vitro, three FAM labeled DNA con- taining regions upstream or downstream of the BRE-TATA region (Fig. 3A, P1-P3), and three non-labeled DNA harboring base substitutions (Fig. 3A, S4-S6) were prepared and EMSAs were performed. TvFur obviously bound to $82.4 \%$ of the 20-bp DNA fragment P2 (5'-G TATTAAT TAATA TTAATT C-3'; Figs. 3B, C, P2). TvFur recognized a DNA fragment of the tvsod-ogg promoter without the BRE, P3, and bound to $65.6 \%$ of the DNA fragment without BRE (Figs. 3B, C, P3). The binding of TvFur to a tvsod-ogg promoter DNA fragment without the TATA-box, P1, was decreased and TvFur bound to $50.9 \%$ of P1 DNA (Figs. 3B, C, P1). Bound TvFur competed with fivefold excess of non-labeled DNA substituted 5'-GTT to 5'-GGG of P2 DNA (Figs. 3D, E, +S4), however, it could not be affected by the DNA of BRE and/or TATA box mutations (Figs. 3D, E, $+\mathrm{S} 5$, $+\mathrm{S} 6$ ). These results indicate that the BRE and TA repeats in the TATA-box are essential for tvsod-ogg promoter recognition by TvFur. Together with the results of the previous study, ${ }^{13)}$ this suggests that TvFur recognizes these TA repeats.

Bacterial Fur proteins bind to a 19-bp inverted repeat sequence known as the classical Fur box. ${ }^{12)}$ The binding of TvFur to the 5'-G T ATTAAT TA-AT ATTAAT T C-3' sequence of the $t v s o d$-ogg promoter DNA implies that it binds to an inverted repeat array. This repeat unit $5^{\prime}$-ATT AAT-3' is also found in the TvFur binding region of the tvfur promoter 5'-GTT ATT ATG TTT ATA T-AT TAA T-TA G-3'. The two inverted repeat units located in the $t v s o d-o g g$ promoter may allow TvFur to bind to this region.

A fur homolog is conserved in the genome of all known $P y$ robaculum species, and an antisense RNA, designated as $R 2$, co-exists with the fur transcript, which probably decreases Fur levels. ${ }^{18)}$ In Escherichia coli, Fur down-regulates the $\operatorname{sod} A$ gene, which encodes Mn-superoxide dismutase, but up-regulates $\operatorname{sodB}$, which encodes Fe-superoxide dismutase, via re- 
A

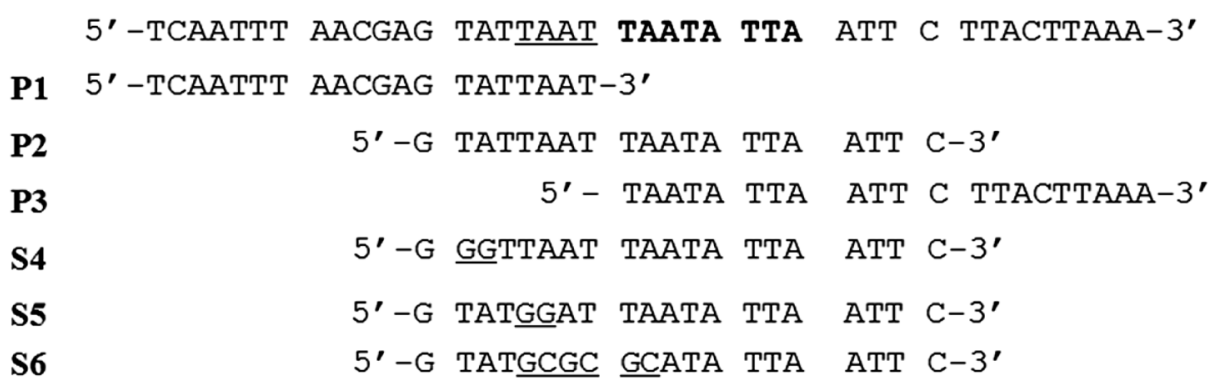

B

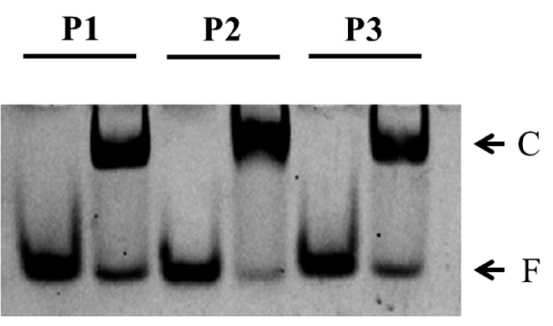

C

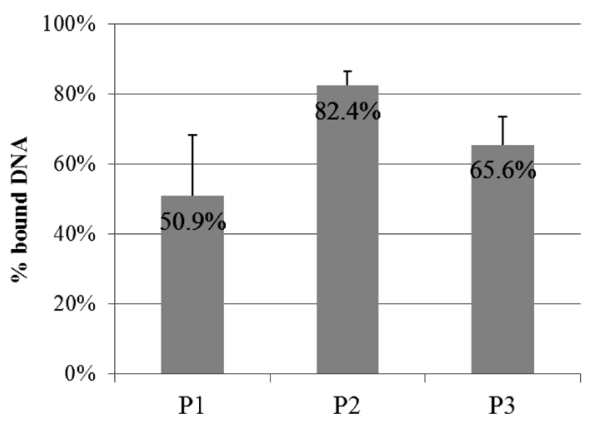

D
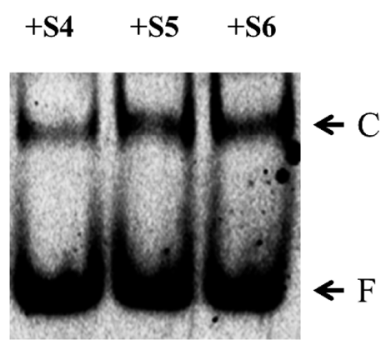

E

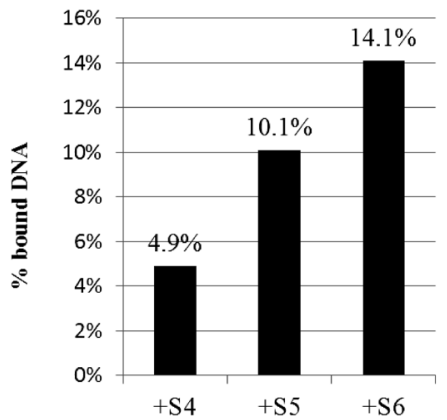

Fig. 3. EMSA Analysis of TvFur Binding to tvsod-ogg Promoter DNA

(A) The sequence of the TvFur binding region in the tvsod-ogg promoter revealed by DNase I footprinting analysis (uppermost sequence, putative BRE and TATA-box are underlined and bolded, respectively), the sequences of the TvFur binding DNA fragments (P1, P2, and P3), and the sequences of DNA fragments containing base substitutions as competitors (S4, S5, and S6, substituted bases were underlined) employed for EMSAs. (B) EMSAs of TvFur binding to 2.0pmol of the DNA fragments shown in Fig. 3A, P1 to P3 in the presence of $100 \mu \mathrm{M} \mathrm{MnCl}_{2}$. EMSA was performed with (right lanes) or without (left lanes) 40 pmol of TvFur. (C) TvFur binding in B is represented as the percentage of bound DNA (indicated in the columns) calculated by subtracting the band intensity of the unbound DNA from that of DNA incubated without TvFur. Error bars represent the S.D. of two independent experiments. (D) EMSA of 4.0 pmol of P2 was performed with 30 pmol of TvFur. Lanes + S4, + S5, and $+\mathrm{S} 6$ contained 30 pmol TvFur and fivefold excess of DNA of S4, S5, and S6 shown in A, respectively. (E) TvFur binding in D is represented as the percentage of bound DNA (indicated above the columns) calculated same as B.

pression of the small RNA RyhB, leading to rapid degradation of the $\operatorname{sodB}$ transcript. ${ }^{19)}$ Several bacterial Fur deletion mutant strains are known to be sensitive to ROS. ${ }^{20-22)}$ These reports indicate that Fur is required for resistance to ROS. This study suggests the possibility that TvFur functions in the same way in Thermoplasma.

\section{REFERENCES}

1) Woese CR, Fox GE. Phylogenetic structure of the prokaryotic domain: the primary kingdoms. Proc. Natl. Acad. Sci. U.S.A., 74, 5088-5090 (1977).

2) Lebedinsky AV, Chernyh NA, Bonch-Osmolovskaya EA. Phylogenetic systematics of microorganisms inhabiting thermal environments. Biochemistry (Moscow), 72, 1299-1312 (2007).

3) Prescott LM, Harley JP, Klein DA. Microbiology, General Topics, Chapter 1-27. The Archaea. McGraw-Hill, New York, pp. 421-437
(1999).

4) Lindahl T. Instability and decay of the primary structure of DNA. Nature, 362, 709-715 (1993).

5) Grollman AP, Moriya M. Mutagenesis by 8-oxoguanine: an enemy within. Trends Genet., 9, 246-249 (1993).

6) Kuchino Y, Mori F, Kasai H, Inoue H, Iwai S, Miura K, Ohtsuka E, Nishimura S. Misreading of DNA templates containing 8-hydroxydeoxyguanosine at the modified base and at adjuscent residues. Nature, 327, 77-79 (1987).

7) Shibutani $S$, Takeshita M, Grollman AP. Insertion of specific bases during DNA synthesis past the oxidation-damaged base 8-oxodG. Nature, 349, 431-434 (1991).

8) Jenney FE Jr, Verhagen MF, Cui X, Adams MW. Anaerobic microbes: Oxygen detoxification without superoxide dismutase. Science, 286, 306-309 (1999).

9) Segerer A, Langworthy TA, Stetter K. Thermoplasma acidophilum and Thermoplasma volcanium sp. nov. from salfatara fields. Syst. Appl. Microbiol., 10, 161-171 (1988). 
10) Sartori AA, Fitz-Gibbon S, Yang H, Miller JH, Jiricny J. A novel uracil-DNA glycosylase with broad substrate specificity and an unusal active site. EMBO J., 21, 3182-3191 (2002).

11) Kaneda K, Ohishi K, Sekiguchi J, Shida T. Characterization of the AP endonucleases from Thermoplasma volcanium and Lactobacillus plantarum: Contributions of two important tryptophan residues to AP site recognition. Biosci. Biotechnol. Biochem., 70, 2213-2221 (2006).

12) Escolar L, Pérez-Martin J, de Lorenzo V. Opening the iron box: Transcriptional metalloregulation by the Fur protein. J. Bacteriol., 181, 6223-6229 (1999).

13) Ikeda $Y$, Minoshima $H$, Satoh $M$, Ishikawa $T$, Kawashima-Ohya Y, Tomobe K, Omata Y, Kawashima T. Transcriptional factor Fur from Thermoplasma volcanium binds its own promoter DNA in a divalent cation-dependent manner. J. Gen. Appl. Microbiol., 58, 465-473 (2012).

14) Hurlburt BK, Yanofsky C. Enhanced operator binding by trp superrepressors of Escherichia coli. J. Biol. Chem., 265, 7853-7858 (1990).

15) Riboulet-Bisson E, Sanguinetti M, Budin-Verneuil A, Auffray Y, Hartke A, Giard JC. Characterization of the Ers regulon of Enterococcus faecalis. Infect. Immun., 76, 3064-3074 (2008).

16) Kawashima $\mathrm{T}$, Amano $\mathrm{N}$, Koike $\mathrm{H}$, Makino $\mathrm{S}$, Higuchi $\mathrm{S}$, Kawashima-Ohya Y, Watanabe K, Yamazaki M, Kanehori K, Kawamoto T, Nunoshiba T, Yamamoto Y, Aramaki H, Makino K,
Suzuki M. Archaeal adaptation to higher temperatures revealed by genomic sequence of Thermoplasma volcanium. Proc. Natl. Acad. Sci. U.S.A., 97, 14257-14262 (2000).

17) Bell SD, Kosa PL, Sigler PB, Jackson SP. Orientation of the transcription preinitiation complex in archaea. Proc. Natl. Acad. Sci. U.S.A., 96, 13662-13667 (1999).

18) Amo T, Atomi H, Imanaka T. Biochemical properties and regulated gene expression of the superoxide dismutase from the facultatively aerobic hyperthermophile Pyrobaculum calidifontis. J. Bacteriol., 185, 6340-6347 (2003).

19) Niederhoffer EC, Naranjo CM, Bradley KL, Fee JA. Control of Escherichia coli superoxide dismutase $(\operatorname{sod} A$ and $\operatorname{sod} B)$ genes by the ferric uptake regulation (fur) locus. J. Bacteriol., 172, 19301938 (1990).

20) Yuhara S, Komatsu H, Goto H, Ohtsubo $Y$, Nagata $Y$, Tsuda M. Pleiotropic roles of iron-responsive transcriptional regulator Fur in Burkholderia multivorans. Microbiology, 154, 1763-1774 (2008).

21) da Silva Neto JF, Braz VS, Italiani VC, Marques MV. Fur controls iron homeostasis and oxidative stress defense in the oligotrophic alpha-proteobacterium Caulobacter crescentus. Nucleic Acids Res., 37, 4812-4825 (2009).

22) Quatrini R, Lefimil C, Veloso FA, Pedroso I, Holmes DS, Jedlicki E. Bioinformatic prediction and experimental verification of Furregulated genes in the extreme acidophile Acidithiobacillus ferrooxidans. Nucleic Acids Res., 35, 2153-2166 (2007). 